\title{
Efecto de la administración de cadmio en las concentraciones de zinc, calcio y hierro en sangre y órganos en ratas macho.
}

\author{
A. Lourdes Castillo Granada ${ }^{a^{*}}$, Maricela Arteaga Mejía ${ }^{b}$ y Rosa Elba Galván Duarte ${ }^{c}$. \\ ${ }^{a}$ Laboratorio de Espectroscopia. \\ ${ }^{b}$ Laboratorio de Proyectos Ambientales. \\ ${ }^{c}$ Unidad de Investigación en Gerontología, Facultad de Estudios Superiores, Zaragoza UNAM. \\ *lourdes_c_g@hotmail.com
}

Recibido 14 Octubre 2013, Aceptado 24 Octubre 2013

\section{Resumen}

La historia de la humanidad ha estado ligada al uso e industrialización de los metales pesados, cuya manipulación sin control ha representado una forma de contaminación antropogénica. Los metales son agentes contaminantes que se difunden con facilidad a través del medio ambiente, y llegan al ser humano principalmente a través del aire, agua y alimentos. La contaminación por causa de los metales pesados es un motivo de preocupación por los posibles daños en la salud. El cadmio es un tóxico peligroso, con una vida media de 10 hasta 30 años, por lo que los síntomas severos pueden presentarse tiempo después de la exposición al metal. La similitud química con elementos esenciales como el zinc y calcio tiene como consecuencia una competencia por las rutas metabólicas. En el presente estudio la administración de cloruro de cadmio a ratas Wistar macho originó una disminución en la concentración de zinc en riñón, hueso y suero sanguíneo.

Palabras clave: Cadmio, metales esenciales, metales pesados, bioacumulación de metales

\section{Introducción}

El tema de la contaminación por metales se ha convertido en un problema vital para la humanidad, debido a la gravedad en sus efectos por causar importantes trastornos ambientales y daños a la salud por acumulación en diferentes órganos y por alteración del equilibrio iónico en el organismo [1, 2, 3].

El cadmio (Cd) es un metal pesado que se encuentra frecuentemente ligado a zinc $(\mathrm{Zn})$ y plomo $(\mathrm{Pb})$. Debido al consumo de productos en los cuales se puede encontrar como contaminante, aunado al hecho de que no se conoce algún efecto benéfico en el organismo y por el contrario presenta daño en riñón, hígado y hueso principalmente, hace que el ingreso al organismo de este metal sea motivo de gran preocupación. $\mathrm{El} \mathrm{Cd}$ fue descubierto en 1817 por Friedrich Strohmeyer, la extracción y el procesamiento de $\mathrm{Zn} \mathrm{y} \mathrm{Pb}$ ocasiona contaminación ambiental por $\mathrm{Cd}$ [4]. Es un tóxico peligroso, la vida media es de 10-30 años, los síntomas severos pueden presentarse tiempo después de la exposición al metal $[5,6]$. En alimentos se encuentra principalmente en mariscos, hígado y riñón con concentraciones mayores a $0.05 \mu \mathrm{g} / \mathrm{g}$ [7].

Desde que se descubrió el cadmio hasta hace 50 años se le encontraron aplicaciones metalúrgicas, se emplea en galvanoplastia, galvanostegia y galvanización, así como en plásticos, pigmentos para pintura (amarillo cadmio), baterías de níquel-cadmio. Presenta gran resistencia a la corrosión. El seleniuro de cadmio (CdSe) se utiliza como pigmento, en celdas solares y películas fotográficas, el sulfato de cadmio se emplea como fungicida [8].

El cadmio se encuentra con valencia $2^{+}$, no forma compuestos alquilo estables ni otros compuestos organometálicos de importancia en toxicología. Se conoce que sus efectos tóxicos resultan de las interacciones con elementos esenciales dentro de los que destaca el zinc; estas interacciones se llevan a cabo en las funciones biológicas de absorción, distribución y excreción, lo que puede interferir con varios procesos metabólicos [9]. La capacidad del organismo para responder a una exposición de cadmio es limitada, el metal no puede sufrir una degradación metabólica, además es pobremente excretado, haciendo que se almacene en órganos blanco por largo tiempo y se convierta en un elemento tóxico. La administración de zinc reduce o elimina los efectos adversos del cadmio [10].

Estudios en animales de laboratorio señalan una absorción de 1.5 por ciento, y en humanos alrededor del 5 por ciento. La absorción por vía respiratoria parece ser más completa, quienes fuman pueden absorber del $10 \mathrm{al}$ 40 por ciento del cadmio inhalado, mientras que otros estudios indican valores mayores al 90 por ciento. Una vez absorbido el cadmio se transporta por la sangre, ligado principalmente a células hemáticas y albúmina. Llega en primer término al hígado, para ser redistribuido lentamente hacia los riñones, en forma del complejo cadmio-metalotioneína (Cd-MT). Después de la distribución, cerca de 50 por ciento de la carga total se distribuye en hígado y riñones [11].

El cadmio es excretado en la orina, bilis y heces. Pequeñas cantidades son eliminadas en el cabello. De modo global, la eliminación de cadmio por heces es cuantitativamente más importante que la excreción del metal por la orina [12].

Cuando es inyectado acetato o cloruro de cadmio en animales de laboratorio se han presentado lesiones en hígado, testículo, hueso, páncreas, pulmón, riñón, corazón, cerebro, sistema inmune y hematopoyético cambiando su morfología, así como daños en placenta, 
mismos que provocan cambios en $\mathrm{ADN}$, proteínas, necrosis y muerte del feto. Algunos investigadores describen un efecto letal en una sola administración de cadmio en ratones y ratas. $\mathrm{La}^{\mathrm{L}} \mathrm{LD}_{50}$ depende de la vía de administración, la edad, especie y el sexo [10].

El $\mathrm{Zn}$ es componente de varias enzimas que intervienen en las principales vías metabólicas, es un cofactor aproximadamente de 200 metaloenzimas en los organismos vivos. Desempeña un papel importante en el sistema de defensa antioxidante. Varias evidencias sugieren que una consecuencia de la deficiencia de zinc puede ser un incremento en el daño oxidativo en tejidos por especies reactivas de oxígeno (ERO). Una deficiencia de zinc puede resultar en tasas altas de daño oxidativo a los lípidos de la membrana, proteínas y en al $\mathrm{ADN}$. Se ha reportado que la deficiencia de zinc altera la maduración de las células espermátidas y provoca daño en los túbulos seminiferos, así como de lesiones testiculares, efecto reversible con la administración del elemento [13]. La concentración de zinc en plasma seminal muestra valores más altos en hombres fértiles comparados con hombres infértiles [14].

El calcio es requerido para la formación de huesos y dientes. Si la dieta es deficiente en este elemento, puede haber problemas óseos y/o dentales. El requerimiento de iones calcio es necesario en la coagulación sanguínea. Con la edad, la constante remoción de calcio del hueso sin suficiente reemplazo, produce el desarrollo gradual de diversos grados de osteoporosis con aumento de fragilidad y fracturas de huesos, en particular de las extremidades y la pelvis. El calcio participa en la excitabilidad nerviosa y muscular, la coagulación sanguínea, la mediación de respuestas hormonales [14].

En los mamíferos, el calcio es importante como iniciador de la reacción del acrosoma en los espermatozoides, existiendo evidencias de que el $\mathrm{Ca}^{2+}$ está involucrado en la movilidad espermática. Además se encuentra en alta concentración en la próstata, vesículas seminales y epidídimo. La concentración del Ca seminal entre hombres fértiles e infértiles presenta diferencias significativas, siendo menor en el grupo de infértiles [15].

En el presente estudio se analizó el comportamiento de los elementos calcio, zinc, y hierro como consecuencia de la ingesta de cadmio. Se cuantificaron los niveles de concentración de $\mathrm{Cd}, \mathrm{Zn}$ y $\mathrm{Ca}$ en cerebro, hígado, riñón, bazo y hueso y en suero sanguíneo.

\section{Parte experimental}

\section{Administración de cloruro de cadmio}

El experimento se realizó en 12 ratas Wistar machos, con un peso aproximado de $200 \pm 20 \mathrm{~g}$, con una edad promedio de ocho semanas. Durante el tratamiento, las ratas permanecieron en el bioterio, bajo condiciones controladas de aire acondicionado y luz (12 horas de luz y 12 horas de oscuridad), se les administró comida y agua ad libitum. Se formaron dos grupos de seis especímenes. Un grupo se consideró como control y al segundo grupo se les administró $15 \mathrm{mg}$ /día de cloruro de cadmio en el agua de beber durante un periodo de 30 días.

\section{Preparación de las muestras}

$\mathrm{Al}$ término del tratamiento, las ratas fueron anestesiadas con éter, se les realizó un corte veno-plexoaxilar obteniendo la sangre en tubos de ensaye, uno con heparina para la cuantificación de cadmio por el método de quelación-extracción con pirrolidin ditiocarmabato de amonio y metilisobutil cetona. Se colectó un segundo tubo sin heparina para la cuantificación de calcio, zinc y hierro en suero sanguíneo. Posteriormente se realizó la disección de los órganos: cerebro, riñón, hígado, bazo y hueso; se pesaron y colocaron en tubos de ensaye, se etiquetaron y se conservaron en refrigeración hasta su procesamiento.

A las muestras de tejido se les realizó una digestión con ácido nítrico, con calentamiento; hasta oxidación completa de la materia orgánica, una vez terminada la digestión se llevó la solución a un matraz aforado de 25 $\mathrm{mL}$, se llevó al volumen con agua desionizada, la solución se filtró y se almacenó en envases de plástico (previamente tratados con ácido nítrico y agua desionizada). Esta solución se emplea para la cuantificación de cadmio, zinc y calcio por espectrofotometría de absorción atómica con llama, empleando aire como oxidante y acetileno como combustible y el sistema de introducción de muestra nebulizador-quemador de flujo laminar.

\section{Cuantificación de calcio, zinc y hierro en suero sanguíneo}

La cuantificación de calcio se realizó en una disolución del suero con de óxido de lantano al 0.1 por ciento (1:25). La determinación de zinc y hierro se realizó en suero diluido con agua (1:5). Las muestras se analizaron por espectrofotometría de absorción atómica. Las preparaciones de referencia y el blanco de reactivos fueron tratados bajo las mismas condiciones.

\section{Resultados y discusión}

Cuadro 1. Concentraciones de $\mathrm{Cd}, \mathrm{Zn}$ y $\mathrm{Ca}$ en riñón determinadas después de 30 días de administración de cloruro de cadmio.

\begin{tabular}{lccc}
\hline Grupo & $\mathrm{Cd}(\mu \mathrm{g} / \mathrm{g})^{\dagger}$ & $\mathrm{Zn}(\mu \mathrm{g} / \mathrm{g}){ }^{\dagger}$ & $\mathrm{Ca}(\mu \mathrm{g} / \mathrm{g})$ \\
\hline Control & $\mathrm{ND}$ & $228.01 \pm 13.83$ & $91.09 \pm 13.55$ \\
Intoxicado & $8.72 \pm 0.44$ & $187.01 \pm 7.83$ & $93.62 \pm 13.22$ \\
\hline$\dagger$ diferencia significativa $\mathrm{P}>0.05$ & \\
ND No detectado.
\end{tabular}

Cuadro 2. Concentraciones de $\mathrm{Cd}, \mathrm{Zn}$ y $\mathrm{Ca}$ en bazo determinadas después de 30 días de administración de cloruro de cadmio.

\begin{tabular}{lccc}
\hline Grupo & $\begin{array}{c}\mathrm{Cd} \\
(\mu \mathrm{g} / \mathrm{g})^{\dagger}\end{array}$ & $\mathrm{Zn}(\mu \mathrm{g} / \mathrm{g})^{\dagger}$ & $\mathrm{Ca}(\mu \mathrm{g} / \mathrm{g})$ \\
\hline Control & $\mathrm{ND}$ & $211.59 \pm 32.50$ & $77.11 \pm 9.28$ \\
Intoxicado & $8.41 \pm 0.43$ & $248.52 \pm 15.76$ & $105.48 \pm 12.45$ \\
\hline
\end{tabular}

$\dagger$ diferencia significativa $\mathrm{P}>0.05$

ND No detectado. 
Cuadro 3. Concentraciones de $\mathrm{Cd}, \mathrm{Zn}$ y $\mathrm{Ca}$ en hígado determinadas después de 30 días de administración de cloruro de cadmio.

\begin{tabular}{lccc}
\hline Grupo & $\mathrm{Cd}(\mu \mathrm{g} / \mathrm{g})^{\dagger}$ & $\mathrm{Zn}(\mu \mathrm{g} / \mathrm{g})^{\dagger}$ & $\mathrm{Ca}(\mu \mathrm{g} / \mathrm{g})^{\dagger}$ \\
& & & \\
\hline Control & $1.01 \pm 0.49$ & $238.52 \pm 27.87$ & $62.37 \pm 8.98$ \\
Intoxicado & $12.13 \pm 1.34$ & $287.01 \pm 38.14$ & $89.29 \pm 7.87$ \\
\hline
\end{tabular}

$\dagger$ diferencia significativa $\mathrm{P}>0.05$

ND No detectado.

Cuadro 4. Concentraciones de $\mathrm{Cd}, \mathrm{Zn}$ y $\mathrm{Ca}$ en cerebro determinadas después de 30 días de administración de cloruro de cadmio

\begin{tabular}{lccc}
\hline Grupo & $\mathrm{Cd}(\mu \mathrm{g} / \mathrm{g})^{\dagger}$ & $\mathrm{Zn}(\mu \mathrm{g} / \mathrm{g})^{\dagger}$ & $\mathrm{Ca}(\mu \mathrm{g} / \mathrm{g})$ \\
& & & \\
\hline Control & $\mathrm{ND}$ & $41.74 \pm 1.87$ & $30.99 \pm 5.08$ \\
Intoxicado & $0.65 \pm 0.19$ & $67.21 \pm 7.89$ & $29.26 \pm 3.91$ \\
\hline
\end{tabular}

$\dagger$ diferencia significativa $\mathrm{P}>0.05$

ND No detectado.

Cuadro 5. Concentraciones de $\mathrm{Cd}, \mathrm{Zn}$ y Ca en hueso después de 30 días de administración de cloruro de cadmio.

\begin{tabular}{lccc}
\hline Grupo & $\mathrm{Cd}(\mu \mathrm{g} / \mathrm{g})^{\dagger}$ & $\mathrm{Zn}(\mu \mathrm{g} / \mathrm{g})^{\dagger}$ & $\mathrm{Ca}(\mu \mathrm{g} / \mathrm{g})$ \\
\hline Control & $0.77 \pm 0.14$ & $724.69 \pm 37.17$ & $869.80 \pm 70.95$ \\
Intoxicado & $4.14 \pm 0.40$ & $154.98 \pm 28.24$ & $849.54 \pm 60.20$
\end{tabular}

$\dagger$ diferencia significativa $\mathrm{P}>0.05$

ND No detectado.

Cuadro 6. Concentraciones de $\mathrm{Cd}, \mathrm{Zn}, \mathrm{Ca}$ y $\mathrm{Fe}$ en suero y sangre completa después de 30 días de administración de cloruro de cadmio.

\begin{tabular}{lcccc}
\hline Grupo & $\begin{array}{c}\mathrm{Cd}^{*} \\
(\mu \mathrm{g} / \mathrm{g})^{\dagger}\end{array}$ & $\begin{array}{c}\mathrm{Zn} \\
(\mu \mathrm{g} / \mathrm{g})^{\dagger}\end{array}$ & $\mathrm{Ca}(\mu \mathrm{g} / \mathrm{g})$ & $\begin{array}{c}\mathrm{Fe} \\
(\mu \mathrm{g} / \mathrm{g})^{\dagger}\end{array}$ \\
\hline Control & $\mathrm{ND}$ & $3.74 \pm 0.2$ & $129.19 \pm 7$. & $25.59 \pm 0$. \\
& & 2 & 85 & 65 \\
Intoxicado & $0.73 \pm 0$ & $2.54 \pm 0.1$ & $123.10 \pm 2$. & $17.23 \pm 0$. \\
& .01 & 0 & 55 & 00 \\
\hline
\end{tabular}

*Sangre completa

$\dagger$ diferencia significativa $\mathrm{P}>0.05$

ND No detectado.

En nuestro estudio encontramos que la administración por 30 días de cloruro de cadmio a ratas macho originó un aumento significativo de la concentración de $\mathrm{Cd}$ en sangre completa, suero sanguíneo y tejidos estudiados. La acumulación de cadmio se asoció con un aumento de la concentración de zinc en el hígado, bazo y cerebro posiblemente como disminución en el proceso de eliminación, la disminución de $\mathrm{Zn}$ en riñón puede deberse a un aumento en el metabolismo depurador de este órgano, para el caso de hueso el efecto puede ser consecuencia de una movilización desde el hueso.

El cadmio es un metal tóxico que principalmente se almacena en riñón e hígado, en este último alcanza su mayor concentración $(12.13 \pm 1.34 \mu \mathrm{g} / \mathrm{g})$, en el presente trabajo también se determinó la concentración de cadmio en cerebro, bazo y hueso.

El cadmio manifiesta su toxicidad en humanos y animales por acumulación en diferentes órganos. El hígado y el riñón son los órganos blanco principales, en los que se encuentra la mayor concentración de Cd. A nivel molecular, el cadmio interfiere con el metabolismo de los metales esenciales como por ejemplo, $\mathrm{Ca}, \mathrm{Zn}, \mathrm{Se}$, $\mathrm{Cr}$ y $\mathrm{Fe}$. La absorción de cadmio a nivel intestinal compite con la absorción de $\mathrm{Zn}$ y $\mathrm{Ca}$, se ha propuesto que el cadmio comparte el mismo sistema de transporte. El Cd inhibe la síntesis de proteínas, el metabolismo de los carbohidratos y las enzimas en el hígado. La exposición ambiental crónica de cadmio produce hipertensión en animales de experimentación [16].

En contraste, el $\mathrm{Ca}$ fue el elemento menos afectado por la ingesta de cloruro de cadmio, con un comportamiento similar al $\mathrm{Zn}$ en bazo, hígado y hueso, es decir con una tendencia a aumentar en el grupo intoxicado.

\section{Conclusiones}

La exposición a $\mathrm{Cd}$ en el modelo murino utilizado generó una disminución en $\mathrm{Zn}$ y Ca en hueso, lo cual podría originar alteraciones en la homeostasis de estos elementos esenciales.

La concentración de $\mathrm{Cd}$ en los órganos estudiados muestra la tendencia de este elemento a acumularse en el organismo.

Las características químicas similares de $\mathrm{Cd}, \mathrm{Zn}$ y Ca puede ser motivo de que compitan en las diferentes rutas metabólicas.

El hierro sufre una disminución considerable en suero como consecuencia de la ingesta de $\mathrm{Cd}$, posiblemente el hecho de ser elementos con valores de electronegatividad similar posibilita la competencia por los sitios activos en el organismo.

\section{Referencias}

1. De Fleurian, G.; Perrin, J.; Ecochard, R.; Dantony, E.; Lanteaume, A.; Achard, V.; Grillo, J.-M.; Guichaoua, M.R.; Botta, A.; Sari-Minodier, I. J Androl 2009, 30, 566.

2. Mathur, N., Pandey, G., \& Jain, G. (2010). Journal of Biological Sciences, 2010, 10(5), 396

3. Sengupta, P. Drug and Chemical Toxicology 2013, 36, 353.

4. Afridi, H. I.; Kazi, T. G.; Jamali, M. K.; Kazi, G. H.; Food and Chemical Toxicology. 2001; 39 (10):967-980.Arain, M. B.; Jalbani, N.; Shar, G. Q.; Sarfaraz, R. A. Toxicology and Industrial Health 2006, 22, 381.1

5. Kowalczyk E, Kopff A, Fijalkowski P, Kopff M, Niedworok J, Blaszczyk J, Kedziora J, Tyslerowicz P. Effect of anthocyanins on selected biochemical parameters in rats exposed to cadmium, Acta Biochim Pol 2003; 50 (2):543-8.

6. Kelley C., Cadmium therapeutic agents. Curr Pharm Des 1999; 5(4): 229-40.

7. Derelanko J, Mannfred A. Handbook of toxicology. $2^{\mathrm{a}}$ ed. Washington: CRC Press; 2002. pp. 890-891, 917-921.

8. Carson B L, Ellis H V. Toxicology and biological monitoring of metals in humans. Chelsea, Michigan: Lewis Publishers Inc; 1986:p.51-58.

9. Reeves, P. G.; Rossow, K. L., The Journal of nutritional biochemistry 1996, 7, 128.

10. Waalkes M P. Cadmium carcinogenesis. Mutation Research/Fundamental and Molecular Mechanisms of Mutagenesis 2003; 533 (1-2): 107-120. 
11. Philp B Richard., Ecosystems and human health toxicology and environmental hazards. Washington: Lewis Publishers; 2001. p. 155-157.

12. Reeves, P. G.; Rossow, K. L., The Journal of nutritional biochemistry 1996, 7, 128.

13. Prasad, A. S. Advances in Nutrition: An International Review Journal 2013, 4, 176

14. Colagar, A. H., Marzony, E. T.; Chaichi, M. J. Nutrition Research 2009, 29, 82.

15. Jimenez-Gonzalez, C.; Michelangeli, F.; Harper, C. V.; Barratt, C. L.; Publicover, S. J. Human Reproduction Update 2006, 12, 253

16. Nath R, Prasad R, Palinal VK, Chopra RK. Prog Food Nutr Sci. 1984; 8(1-2):109-63. 